\title{
Fluctuations of sensorimotor processing in migraine: a controlled longitudinal study of beta event related desynchronization
}

\author{
Martin Syvertsen Mykland ${ }^{1 *}$ (D), Marte Helene Bjørk ${ }^{2,3}$, Marit Stjern ${ }^{1,4}$, Petter Moe Omland ${ }^{1,4}$, Martin Uglem ${ }^{1,4}$ and \\ Trond Sand ${ }^{1,4}$
}

\begin{abstract}
Background: The migraine brain seems to undergo cyclic fluctuations of sensory processing. For instance, during the preictal phase, migraineurs experience symptoms and signs of altered pain perception as well as other wellknown premonitory CNS-symptoms. In the present study we measured EEG-activation to non-painful motor and sensorimotor tasks in the different phases of the migraine cycle by longitudinal measurements of beta event related desynchronization (beta-ERD).
\end{abstract}

Methods: We recorded electroencephalography (EEG) of 41 migraine patients and 31 healthy controls. Each subject underwent three EEG recordings on three different days with classification of each EEG recording according to the actual migraine phase. During each recording, subjects performed one motor and one sensorimotor task with the flexion-extension movement of the right wrist.

Results: Migraine patients had significantly increased beta-ERD and higher baseline beta power at the contralateral C3 electrode overlying the primary sensorimotor cortex in the preictal phase compared to the interictal phase. We found no significant differences in beta-ERD or baseline beta power between interictal migraineurs and controls.

Conclusion: Increased preictal baseline beta activity may reflect a decrease in pre-activation in the sensorimotor cortex. Altered pre-activation may lead to changes in thresholds for inhibitory responses and increased beta-ERD response, possibly reflecting a generally increased preictal cortical responsivity in migraine. Cyclic fluctuations in the activity of second- and third-order afferent somatosensory neurons, and their associated cortical and/or thalamic interneurons, may accordingly also be a central part of the migraine pathophysiology.

Keywords: Migraine, Headache, Cycle, Cortex, Thalamus, EEG, ERD, Pre-activation, Pathophysiology, Neurophysiology

\section{Introduction}

Migraine patients undergo transient clinical and neurophysiological changes before, during and after headache attacks. These intervals define the phases of the migraine cycle, and pain perception changes transiently between the phases [1, 2]. In the days and hours preceding headache, various other symptoms including yawning, nausea, changes in mood and activity, fatigue and neck symptoms emerge [3-9]. Furthermore, neuroimaging

\footnotetext{
* Correspondence: martin.s.mykland@ntnu.no

${ }^{1}$ Department of Neuromedicine and Movement Science, Faculty of Medicine and Health Sciences, NTNU - Norwegian University of Science and Technology, Trondheim, Norway

Full list of author information is available at the end of the article
}

and neurophysiological studies show alterations of the central nervous system (CNS) preceding the eventual aura and the pain [2, 10-20]. Sensitized thalamic neurons may mediate allodynia and hyperalgesia $[8,21]$, and the connectivity between thalamus and several brain regions has also been shown to undergo cyclic changes in migraineurs [22]. However, there is disagreement between different studies regarding whether the migraine brain become hypo- or hyperexcitable [19, 23-25]. Further mapping of the preictal neurophysiological state may reveal the probable CNS generators for the migraine attacks.

One technique for measuring cortical activation and increased excitability during sensorimotor processing is beta event-related desynchronization (beta-ERD) 
[26-28]. Event related desynchronization and synchronization (ERD/ERS) are electrophysiological features that represent an induced, time-locked, nonphase-locked response to events. These responses are subject to changes in neuronal synchrony. Furthermore, they are specific for frequencies in the electroencephalography (EEG). In general the ERD/ERS is understood to represent changes in activity of the interactions between thalamocortical networks and cortical interneurons [29]. The cortical activity accompanying voluntary limb movement is for instance represented by ERD of alpha and beta bands in the contralateral sensorimotor cortex, reflecting cortical activation with enhanced information processing $[27,28]$. More recent knowledge of time averaged betaERD suggest that the strength of the ERD response during hand movements represents mostly the afferent proprioceptive sensation and sensory processing [30]. One study of fibromyalgia patients showed altered beta-ERD in response to tactile stimulation, which was interpreted by the authors as physiological changes that contribute to chronic pain in this patient group [31]. This technique may accordingly be useful for investigating cortical sensory processing in migraine patients.

As the migraine brain is subject to cyclic neurophysiological fluctuations, it is advantageous to conduct longitudinal studies [32]. In a recent paper on postmovement beta synchronization (PMBS) in migraine [14], we revealed cyclic fluctuations of post-stimulation inhibition in sensorimotor cortex. To our knowledge the actual desynchronization during movement has not been analysed previously in migraine. Thus, in the present paper we aimed to measure thalamocortical excitability in migraine patients by cortical beta-ERD during sensory processing of afferent inputs from hand movements. The first primary aim was to compare cyclic changes from the interictal baseline to the preictal, ictal and postictal phases. The second primary aim was to compare interictal beta-ERD between migraineurs and healthy controls. A third, and secondary, aim was to evaluate if these electrophysiological responses correlated with clinical symptoms and severity.

\section{Subjects and methods Subjects}

The general methodology for this study is described in our previous papers $[14,18-20,32,33]$. We recruited episodic migraine patients by a newspaper advertisement, screening by trained nurses, and evaluation for inclusion by a neurologist [32]. They had 2-6 migraine attacks each month. Healthy controls were recruited among blood donors. Exclusion criteria were frequent episodic or chronic tension-type headache, acute or chronic disease, pregnancy, alcohol or drug abuse, neuroactive drug use and migraine prophylactic drugs within four weeks before the test. We included 41 migraine patients and 31 healthy controls. All but 4 patients and 3 controls were right handed.

We recorded demographic data on all subjects in addition to clinical presentation of the migraine patients by a questionnaire and a semi-structured interview (Table 1). Every migraine patient also completed a headache diary from 2 weeks before inclusion until 2 weeks after the last EEG recording. This enabled us to classify the headache and its temporal relationship to the EEG recordings as preictal $(<36 \mathrm{~h}$ before attack), postictal $(<36 \mathrm{~h}$ after attack), ictal (pain attack) and interictal ( $>36 \mathrm{~h}$ from attack). The choice of $36 \mathrm{~h}$ cut off is based on an electronic diary study revealing that reliable premonitory symptoms mainly occurred within a one-day cut off [7], and the utility of the $36 \mathrm{~h}$ cut off used in our previous EEG-studies in migraine [14, 18-20, 32, 33]. Every subject (except one) had three EEG recordings at the same time of day with 3-10 days intervals. Thirtythree patients had at least one interictal recording. Patients with both near-attack and interictal recordings formed three additional migraine subgroups for intraindividual paired analysis (Table 1). Six patients had visual aura, two of whom with additional somatosensory aura.

Staff involved in data recording and EEG-response processing was blinded to the diagnosis status. The subjects received NOK 1000 (about EUR 103 with current exchange rates) as compensation to cover expenses after completing all three recordings. The compensation was not mentioned in the advertisement.

\section{EEG recordings and experimental setup}

We recorded approximately $30 \mathrm{~min}$ EEG with eyes closed. Five minutes undisturbed relaxed wakefulness was followed by a motor and a sensorimotor test and by photic stimulation. We have previously reported resting state quantitative EEG, steady-state visual evoked potentials and PMBS [14, 18, 19, 32, 33].

We attached twenty-four scalp electrodes according to the 10/20 international system [34] with channels for lateral anterior temporal electrodes, horizontal and vertical eye movements, and ECG. EEG was recorded digitally in Nervus 3.0 with M40 amplifier (Natus Medical Inc., Pleasanton, CA 94566, USA) and common reference with 256 $\mathrm{Hz}$ sampling rate. We used an average reference montage with low- and high-pass filter of 0.5 and $70 \mathrm{~Hz}$ in addition to notch filter $(50 \mathrm{~Hz})$. Two EMG-channels for flexion and extension were included in the EEG recording for determination of movement epochs (Fig. 1).

Each subject performed both a motor test $(\mathrm{M})$ and a sensorimotor test (SM) with approximately 30 repeated movements of the right arm in each test. The order of tests was randomized for each subject and fixed for each day of recording for the same subject. The instructions 
Table 1 Demographic and clinical data on groups used in interictal analysis (comparing recordings in interictal migraineurs and controls) and subgroups used in paired analysis

\begin{tabular}{|c|c|c|c|c|}
\hline & & Migraineur & |ysis ${ }^{2}$ & \\
\hline & Interictal recordings ${ }^{1}$ & Preictal & Ictal & Postictal \\
\hline & $(n=33)$ & $(n=11)$ & $(n=13)$ & $(n=9)$ \\
\hline Women/men & $30 / 3$ & $11 / 0$ & $12 / 1$ & $7 / 2$ \\
\hline MwoA/MA & $27 / 6$ & $9 / 2$ & $10 / 3$ & $8 / 1$ \\
\hline Age (years) & $36.5(12.7)$ & $37.3(12.9)$ & $37.5(12.5)$ & $41.3(12.8)$ \\
\hline Headache history (years) & $19.3(11.0)$ & $20.5(11.7)$ & $20.5(9.9)$ & $18.1(13.1)$ \\
\hline Headache days last 3 months & $6.2(4.0)$ & $6.7(4.8)$ & $7.2(4.7)$ & $4.2(2.3)$ \\
\hline Headache intensity (0-4) & $2.4(0.7)$ & $2.4(0.7)$ & $2.3(0.6)$ & $2.2(1.0)$ \\
\hline Headache duration (h) & $17.8(22.0)$ & $15.9(20.2)$ & $14.9(17.3)$ & $18.4(30.5)$ \\
\hline Photophobia $(0-2)^{3}$ & $1.4(0.7)$ & $1.4(0.7)$ & $1.0(0.8)$ & $1.2(0.7)$ \\
\hline Phonophobia $(0-2)^{3}$ & $1.1(0.8)$ & $1.2(0.7)$ & $0.8(0.8)$ & $1.2(0.8)$ \\
\hline
\end{tabular}

$M A=$ migraine with aura, $M w O A=$ migraine without aura. Mean (SD) or numbers ${ }^{1}$ Migraineurs with at least one interictal EEG recording

${ }^{2}$ Subgroups with both an interictal EEG recording ( $>36 \mathrm{~h}$ from attack) and a preictal $(<36 \mathrm{~h}$ before attack), ictal or postictal $(<36 \mathrm{~h}$ after attack) EEG recording

${ }^{3}$ Numerical definition: 0 - none, 1 - some, 2 - substantial

The control group consisted of 27 women and 3 men $(n=30)$ with a mean age of $39.7(11.5)$

given to all subjects were the following: Each test would last about $8 \mathrm{~min}$ with a light blink indicating when to start each movement. For the motor test, subjects were to first flex their wrist for $2 \mathrm{~s}$, then extend their wrist for $2 \mathrm{~s}$, followed by about $15 \mathrm{~s}$ of relaxation. For the sensorimotor test an identical flexion-extension movement supplemented by a discrimination task was performed: A bowl of different material spheres (wood or metal) was placed about $5 \mathrm{~cm}$ below the neutrally positioned fingers so that the fingers were in contact with the spheres in the flexed position. The task was to use the $2 \mathrm{~s}$ in the flexed position to scan spheres lightly with their fingertips to detect if a sphere of wood was present in the bowl or not. The right arm was used in both sequences.

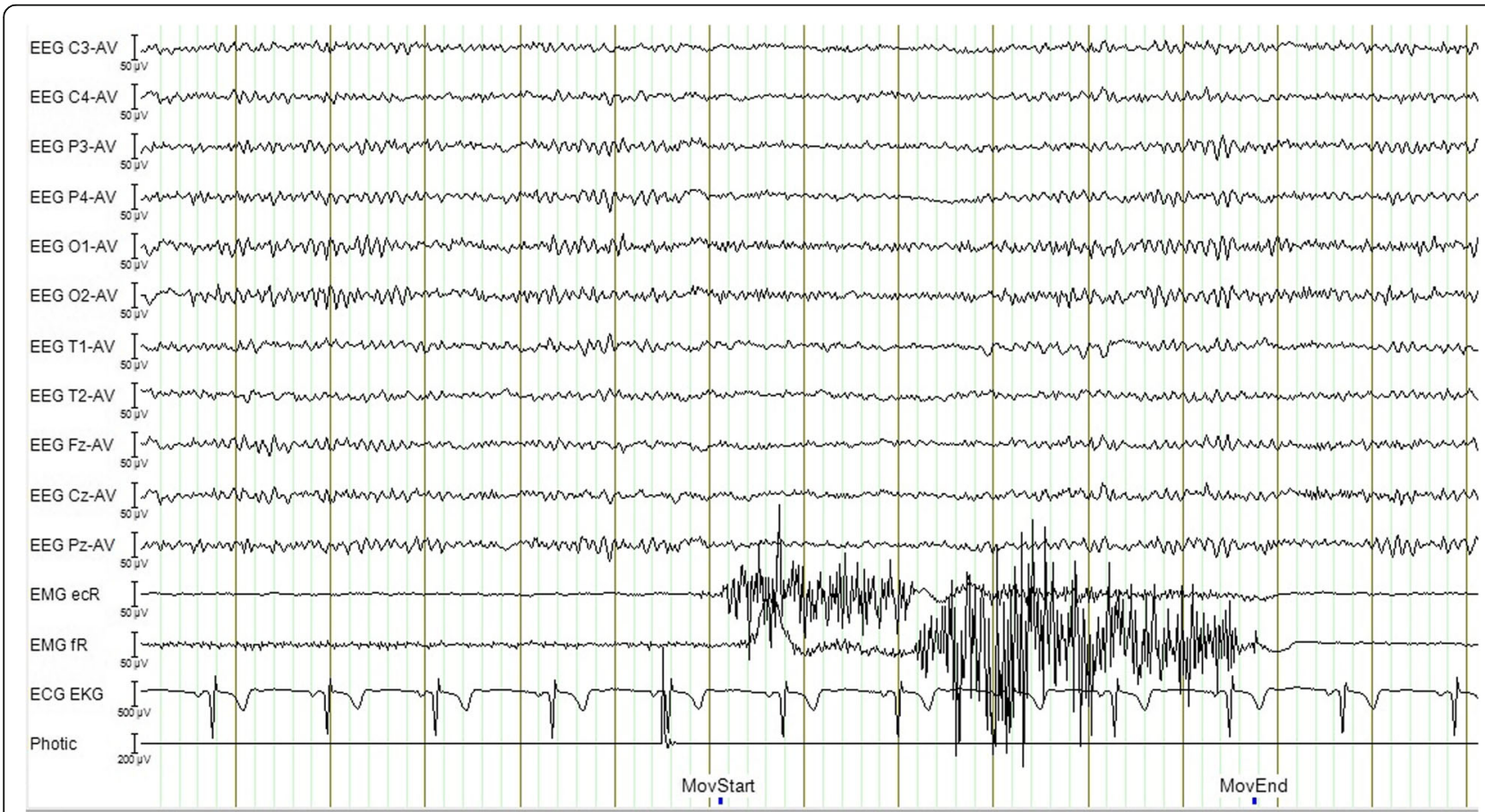

Fig. $1 \mathrm{EEG}, \mathrm{EMG}$ (ecR: radial wrist extensors, fR: forearm wrist flexors), ECG and photic channels from recording of one single movement. MovStart and MovEnd markers indicate start and end of movement as shown by EMG channels. The photic channel marker represents a light blink as a sign for the subject to prepare for executing the task. EEG channels visually revealing desynchronization from start of movement 


\section{ERD analysis}

IIR-filtered data in the $12-19 \mathrm{~Hz}$ beta frequency band $[35,36]$ from sensorimotor cortices electrodes C3 (left side, contralateral) and $\mathrm{C} 4$ (right side, ipsilateral), were exported in $256 \mathrm{~Hz}$ resolution from each test and used for the beta-ERD analysis. Voluntary movement intent lowers beta band activity close to the contralateral sensorimotor cortical area, and this beta-ERD spreads and becomes bilateral right before movement execution $[29,37,38]$. We averaged the squared EEG-amplitude across all movements within the same test [29]. Movement onset and offset were marked (Fig. 1). We used the EEG-segment from $-3 \mathrm{~s}$ to $-1 \mathrm{~s}$ (prior to start of movement) as baseline and the EEG-segment from $1 \mathrm{~s}$ to $3 \mathrm{~s}$ after movement onset for ERD-calculation as established in previous studies [27, 31]. ERD was thereafter calculated as the ratio of power in the activity period to power in the baseline period [27]. ERD can be observed as reduced EEG-amplitude (Fig. 1) and as reduced betapower during movement (Fig. 2).

\section{Statistical analysis}

For each migraine patient we selected one test for each cyclic phase that was available (interictal, preictal, ictal and postictal). If several tests for the same phase were available, we chose the second one. We selected control EEGs with a similar test-order distribution as the interictal migraine group.

We used paired Student's t-tests for the three primary pre-planned intrasubject contrasts. Two-sided $p$-values $<0.05$ were regarded as significant, and p-values $<0.10$ were regarded as trends. A paired analysis with 11 pairs has approximately $77 \%$ power to detect an effect $=90 \%$ of group SD [32].

To evaluate the combined effects of "phase", "task" and "side" factors, we also performed three separate repeated measures ANOVAs (R-ANOVA) within the migraine group (preictal-interictal, ictal-interictal and postictal-interictal differences respectively). Three within-subject factors were specified; "phase", "side" (C3 vs $\mathrm{C} 4$ ) and "SM/M" (sensorimotor vs motor test).

Similarly, to evaluate differences between controls and migraineurs in the interictal phase, we used two sample Student's t-test followed by R-ANOVA on LNtransformed ERD-ratios. We used within-subject factors "side" (C3 vs C4) and "SM/M" (sensorimotor vs motor test), and between-subjects factor "group" (CO vs MIG).

We calculated exploratory Spearman rho correlations for headache history duration, usual attack duration, individual scores on headache frequency $(0-4)$, usual headache intensity (0-4), individual scores on photophobia (0-2) and individual scores on phonophobia (0-2), and beta-ERD.

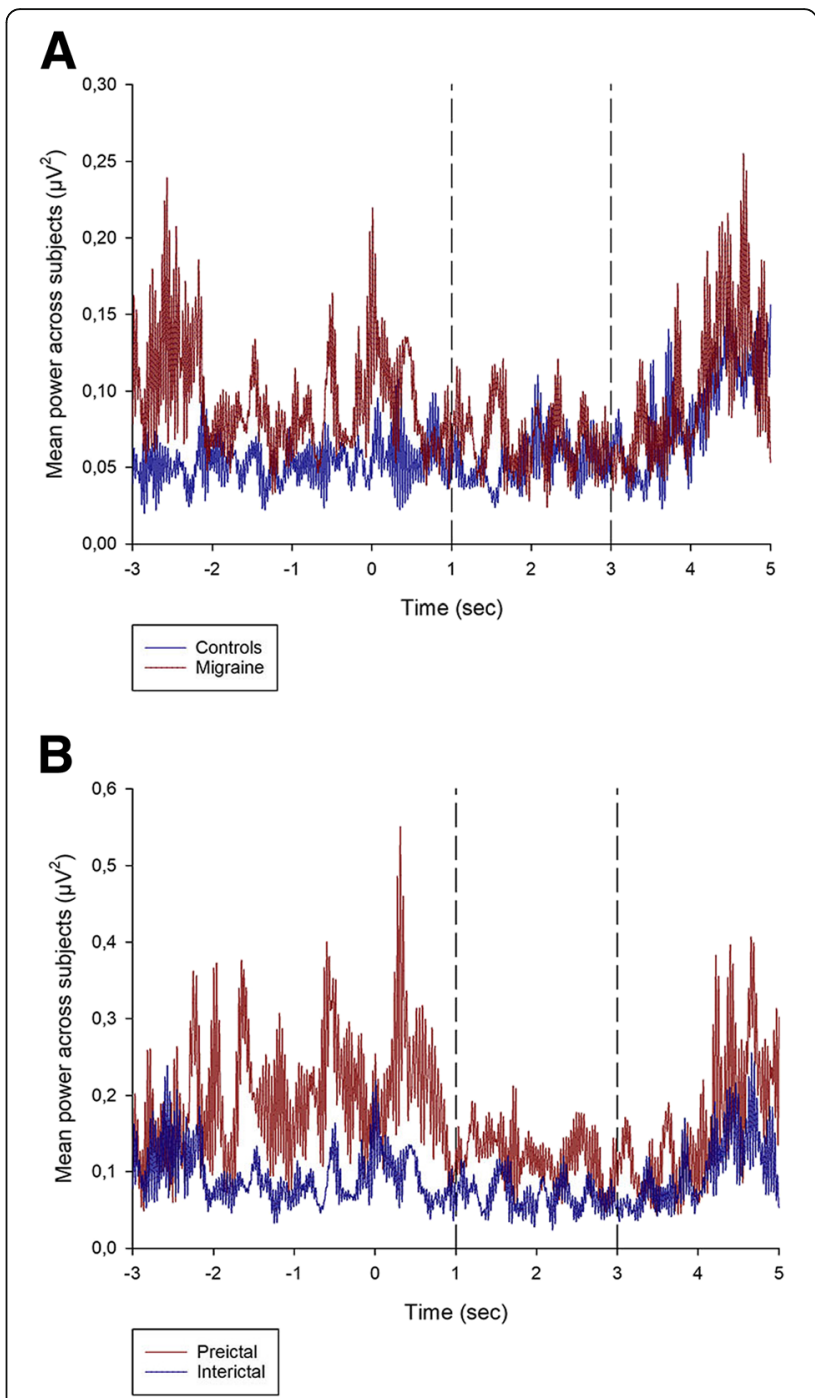

Fig. 2 a Grand mean power across subjects at the contralateral C3 electrode for the sensorimotor task in controls and interictal migraine patients. b Grand mean power across subjects at the contralateral C3 electrode for the sensorimotor task in migraine patients for interictal and preictal recordings. First two seconds ( -3 to -1 ) represent pre-movement onset baseline. 0 represent start of movement. Broken vertical lines indicate the selected interval (1 to $3 \mathrm{~s}$ ) for the ERD period

\section{Results}

Responses were generally larger (lower ratios) for SM than for M-tasks (Table 2), and the task-factor was significant both in preictal and ictal phases (Table 3).

\section{Paired analyses for preictal, ictal and postictal phases compared to the interictal period}

Paired comparison (Table 2) revealed significantly increased beta-ERD responses in contralateral sensorimotor cortex (C3) in the preictal phase compared to the interictal phase for both the SM and M-tasks (C3 SM, $p=0.038$; C3 M, $p=0.049$; Fig. 3). However, R-ANOVA 
Table 2 Beta-ERD responses for preictal-interictal, ictal-interictal and postictal-interictal comparisons

\begin{tabular}{llll}
\hline Beta-ERD response & \multicolumn{4}{l}{$l$} \\
\hline C3 Sensorimotor & Interictal & Compared period & $p$ \\
Preictal & $0.84(0.61-1.15)$ & $0.71(0.46-1.10)$ & .038 \\
Ictal & $0.79(0.62-1.01)$ & $0.74(0.54-1.02)$ & .49 \\
Postictal & $0.74(0.49-1.12)$ & $0.73(0.54-1.00)$ & .97 \\
C3 Motor & & & \\
Preictal & $1.12(0.78-1.61)$ & $0.96(0.76-1.21)$ & .049 \\
Ictal & $1.05(0.72-1.52)$ & $1.04(0.69-1.55)$ & .93 \\
Postictal & $0.93(0.49-1.74)$ & $0.73(0.47-1.14)$ & .23 \\
C4 Sensorimotor & & & .23 \\
Preictal & $0.83(0.65-1.06)$ & $0.71(0.48-1.05)$ & .70 \\
Ictal & $0.83(0.56-1.21)$ & $0.79(0.51-1.24)$ & .72 \\
Postictal & $0.68(0.46-1.02)$ & $0.72(0.57-0.92)$ & \\
C4 Motor & & & .76 \\
Preictal & $1.10(0.70-1.70)$ & $1.14(0.74-1.74)$ & .73 \\
Ictal & $1.19(0.76-1.85)$ & $1.23(0.82-1.86)$ & .23 \\
Postictal & $1.06(0.57-1.99)$ & $0.76(0.44-1.34)$ & \\
\hline
\end{tabular}

Beta-ERD response is the ratio between mean power in the interval from 1 to $3 \mathrm{~s}$ after movement onset and mean power in the interval -3 to $-1 \mathrm{~s}$ before movement onset (baseline). Ratios were LN-transformed before statistical analysis and retransformed to mean ratios and mean \pm SD for tabulation. Paired Student's t-tests are included. EEG from central electrodes C3 (contralateral, left) and C4 (ipsilateral right) for the sensorimotor test and motor test

Bold entries are significant $p$-values

Table 3 Repeated measures ANOVA beta-ERD paired analysis in different phases of the migraine cycle (preictal, ictal and postictal; compared to a paired interictal recording)

\begin{tabular}{|c|c|c|c|c|c|c|}
\hline \multicolumn{7}{|l|}{ Beta-ERD response } \\
\hline \multirow[t]{2}{*}{ Within subjects effects } & \multicolumn{2}{|l|}{ Preictal } & \multicolumn{2}{|l|}{ Ictal } & \multicolumn{2}{|l|}{ Postictal } \\
\hline & $F(1,10)$ & $p$ & $F(1,12)$ & $p$ & $F(1,8)$ & $p$ \\
\hline Phase & 3.472 & .089 & .045 & .84 & .833 & .39 \\
\hline Side & .319 & .58 & 3.318 & .094 & .162 & .70 \\
\hline SM/M & 6.084 & .031 & 18.706 & .001 & 2.623 & .14 \\
\hline Side $\times$ SM $/ M$ & 1.231 & .29 & 3.448 & .088 & 3.156 & .11 \\
\hline Side $\times$ Phase & 3.105 & .11 & .533 & .48 & .094 & .77 \\
\hline SM/M $\times$ Phase & .539 & .48 & .305 & .59 & 1.721 & .23 \\
\hline Side $\times$ SM $/ M \times$ Phase & 2.533 & .14 & .125 & .73 & 2.072 & .19 \\
\hline
\end{tabular}

Paired analysis of subgroups preictal ( $<36 \mathrm{~h}$ before migraine pain attack), ictal and postictal ( $<36 \mathrm{~h}$ after migraine pain attack). Beta-ERD response is the ratio between mean power in the interval from 1 to $3 \mathrm{~s}$ after movement onset and mean power in the interval -3 to $-1 \mathrm{~s}$ before movement onset (baseline). Ratios were LN-transformed before statistical analysis. Factors used were side (C3 vs C4 electrode), SM/M (sensorimotor vs motor) and cyclic phase (preictal-interictal, ictal-interictal and postictal-interictal)
(Table 3) revealed only a trend towards a difference between the interictal and preictal phase $(p=0.089)$ presumably because the C4-response was similar in preictal and interictal phases (Table 2). Furthermore, paired Student's t-tests for baseline (Table 4) revealed significantly increased beta power in contralateral sensorimotor cortex $(\mathrm{C} 3)$ in the preictal phase compared to the interictal phase for both the SM and M-tasks (C3 SM, $p=0.007$; C3 $\mathrm{M}, p=0.048)$. We also discovered significantly increased baseline beta power (Table 4) in ipsilateral sensorimotor cortex (C4) in the ictal phase compared to the interictal phase for the SM test (C4 SM, $p=0.009$ ).

Interictal analyses between migraine patients and controls Contralateral beta-ERD was more evident for the SMtask than for the M-task, both in migraine (SM test ratio 0.79 ) and controls (SM test ratio 0.82; Table 5). This was also shown by a significant effect of $S M / M$ in the RANOVA analysis $(p<0.001$; Table 6 and Fig. 3). RANOVA confirmed no overall difference in beta-ERD between migraine patients and controls (Table 6). For both groups we observed a generally stronger beta-ERD at the contralateral side $(\mathrm{C} 3)$ revealed by a significant effect of side (Table 6). We found no significant differences in baseline beta power between interictal migraineurs and controls.

\section{Relationship between beta-ERD and clinical variables}

In order to limit the number of estimations, correlation coefficients were only calculated from contralateral betaERD values in the interictal and preictal phases. No significant correlations were found for these variables. A trend was seen towards a positive correlation between preictal contralateral (C3) beta-ERD in the M-task and headache intensity ( $r h o=0.41, p=0.095$ ), and towards a negative correlation between interictal contralateral (C3) beta-ERD in the SM-task and headache history (rho $=-0.32, p=0.076$ ).

\section{Discussion}

The main result in this study was increased beta-ERD in the preictal phase compared to the interictal phase over contralateral sensorimotor cortex. This finding suggests that cortical processing of sensorimotor input changes during $36 \mathrm{~h}$ before the headache attack in migraine. Based on current understanding of the beta-ERD property during hand movement [27, 28, 30], the findings suggest increased sensorimotor cortex excitability in the preictal phase compared to the interictal phase. Another important finding was the larger preictal baseline beta activity in migraine, possibly representing lower sensorimotor cortex pre-activation in the preictal phase. We did not find this central sensory fluctuation during the ictal phase, but the study was not powered to perform 


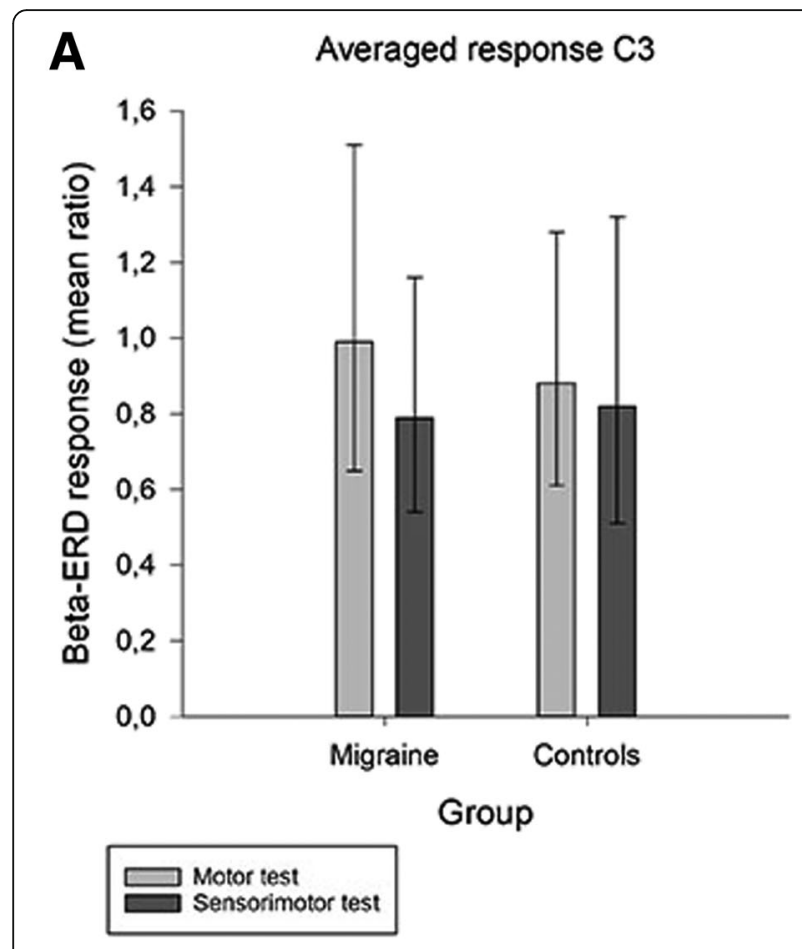

\section{B Averaged response $\mathrm{C} 3$ sensorimotor test}

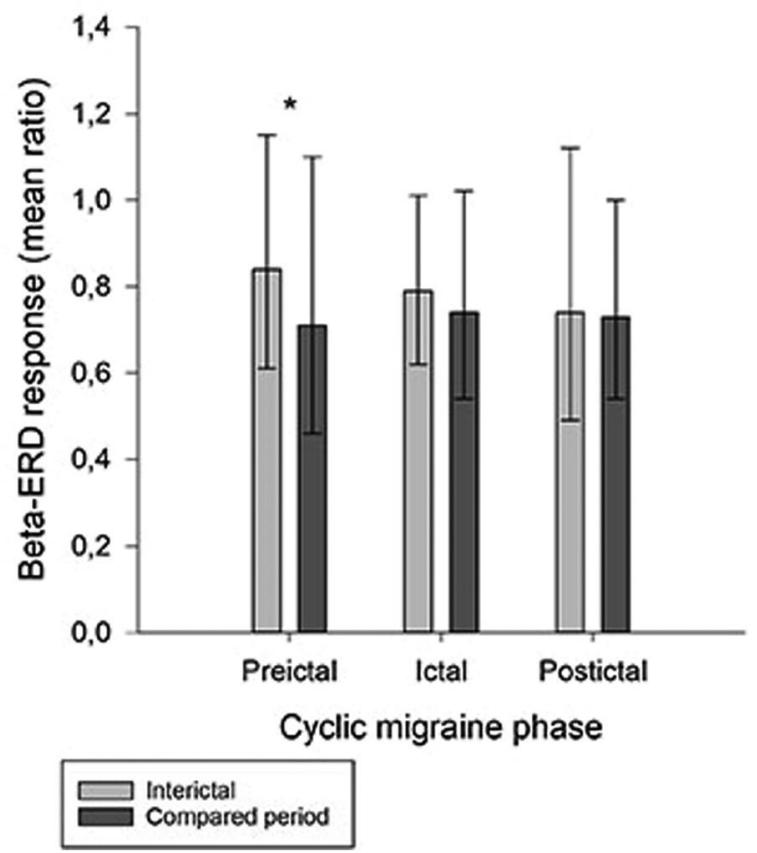

Fig. 3 Beta-ERD ratios (movement interval divided by baseline interval) for contralateral sensorimotor cortex (C3) with lower ratios indicating increased ERD-response. Error bars are mean \pm SD (retransformed). a The figure is illustrating generally larger beta-ERD response for sensorimotor test than for motor test (significant effect of SM/M in the R-ANOVA analysis $(p<0.001)$. $\mathbf{b}$ Beta-ERD was significantly increased preictally $(p=0.038)$
Table 4 Baseline beta power for preictal-interictal, ictal-interictal and postictal-interictal

\begin{tabular}{llll}
\hline Interval mean & Interictal & Compared period & \\
\hline C3 Sensorimotor & & & $\mathrm{p}$ \\
Preictal & $1.27(1.05-1.50)$ & $1.34(1.08-1.59)$ & $\mathbf{. 0 0 7}$ \\
Ictal & $1.23(1.05-1.41)$ & $1.24(1.03-1.46)$ & .696 \\
Postictal & $1.23(0.99-1.46)$ & $1.21(1.08-1.35)$ & .802 \\
C3 Motor & & & \\
Preictal & $1.28(1.07-1.49)$ & $1.33(1.08-1.58)$ & $\mathbf{. 0 4 8}$ \\
Ictal & $1.25(1.07-1.42)$ & $1.25(1.06-1.44)$ & .868 \\
Postictal & $1.26(1.01-1.50)$ & $1.25(1.04-1.45)$ & .692 \\
C4 Sensorimotor & & & \\
Preictal & $1.31(1.09-1.54)$ & $1.34(1.07-1.62)$ & .335 \\
Ictal & $1.23(1.03-1.43)$ & $1.28(1.05-1.50)$ & .009 \\
Postictal & $1.26(1.04-1.49)$ & $1.25(1.10-1.39)$ & .692 \\
C4 Motor & & & .637 \\
Preictal & $1.32(1.10-1.55)$ & $1.34(1.09-1.59)$ & .648 \\
Ictal & $1.27(1.07-1.46)$ & $1.28(1.05-1.50)$ & .665 \\
Postictal & $1.29(1.05-1.53)$ & $1.28(1.04-1.51)$ & .69 \\
\hline
\end{tabular}

Beta power to the 0.25 th power in the interval -3 to $-1 \mathrm{~s}$ before movement onset (baseline). Tabulated as mean \pm SD. Post-hoc paired Student's t-tests are included. EEG from central electrodes C3 (contralateral, left) and C4 (ipsilateral, right) for the sensorimotor test and motor test

direct comparisons between the preictal and the ictal phase. Moreover, we did not discover any significant differences in baseline beta power or beta-ERD between migraineurs in the interictal phase and control subjects.

We observed generally larger strength of ERD during the sensorimotor test than during the pure motor test. The sensorimotor process measured as beta-ERD is averaged over time and consists of several sub-processes from different neuronal networks including motor intention, motor planning, command generation and sensory feedback. To execute movement over time it is necessary to maintain motor processing of proprioceptive sensory feedback. Studies on beta-ERD have shown

Table 5 Beta-ERD mean of response/baseline ratios in interictal migraine subjects and controls

\begin{tabular}{lllllll}
\hline Beta-ERD response & \multicolumn{2}{l}{ Mean ratio $( \pm$ SD retransformed) } & & & \\
\cline { 2 - 5 } & Migraine & Control & t & df & P \\
\hline C3 SM & $0.79(0.54-1.16)$ & $0.82(0.51-1.32)$ & .30 & 57.4 & .76 \\
C3 M & $0.99(0.65-1.51)$ & $0.88(0.61-1.28)$ & -1.12 & 61.8 & .27 \\
C4 SM & $0.77(0.53-1.13)$ & $0.87(0.58-1.31)$ & 1.21 & 60.6 & .23 \\
C4 M & $1.05(0.63-1.74)$ & $1.07(0.70-1.64)$ & .14 & 61.3 & .89
\end{tabular}

Beta-ERD response is the ratio between mean power in the interval from 1 to $3 \mathrm{~s}$ after movement onset and mean power in the interval -3 to $-1 \mathrm{~s}$ before movement onset (baseline). Ratios were LN-transformed before statistical analysis and retransformed to mean ratios and mean \pm SD for tabulation. Two-sample Student's t-tests (equal variance not assumed) are included. EEG from central electrodes C3 (contralateral, left) and C4 (ipsilateral, right). $S M=$ sensorimotor test, $M=$ motor test 
Table 6 Repeated measures ANOVA beta-ERD analysis of interictal migraine patients compared to controls

\begin{tabular}{lll}
\hline & \multicolumn{2}{l}{ Beta-ERD response } \\
\cline { 2 - 3 } Within subjects effect & $\mathrm{F}(1,61)$ & $\mathrm{p}$ \\
\hline Side & 7.24 & $\mathbf{. 0 0 9}$ \\
SM/M & 14.65 & $<.001$ \\
Side $\times$ SM/M & 10.27 & $\mathbf{. 0 0 2}$ \\
Side $\times$ Group & 3.94 & $\mathbf{. 0 5 2}$ \\
SM/M $\times$ Group & 1.36 & .25 \\
Side $\times$ SM/M $\times$ Group & .40 & .53 \\
Between subjects effect & $\mathrm{F}(1,61)$ & $\mathrm{p}$ \\
Group & .027 & .87 \\
\hline
\end{tabular}

Beta-ERD response is the ratio between mean power in the interval from 1 to $3 \mathrm{~s}$ after movement onset and mean power in the interval -3 to $-1 \mathrm{~s}$ before movement onset (baseline). Ratios were LN-transformed before statistical analysis. Within subject factors used were side (C3 vs C4 electrode) and SM/M (sensorimotor vs motor task). The between subjects factor is Group (interictal migraine vs controls)

the motor command-generating process to have little effect on the ERD response. Instead, ERD seems to reflect variation in proprioceptive sensation [30]. This theory might explain why the ERD response was larger for the sensorimotor task compared to the motor task. Accordingly, the observed cyclic fluctuation of beta-ERD in migraineurs may represent a relative change in central sensory processing.

The present results can be largely explained as an increased contralateral sensorimotor cortical response to movement in the preictal phase compared to the interictal phase. Increased ERD response may reflect increased cortical excitability. However, increased baseline beta power suggests increased preictal intracortical inhibition and/or less preictal thalamocortical activation prior to stimuli. The findings therefore indicate that cortical excitation during the task is increased relative to an altered baseline. We interpret this as reduced sensorimotor cortical pre-activation in the preictal phase.

Reduced pre-activation was originally proposed to reflect reduced serotenoergic "state setting" in order to explain intensity-dependence of auditory evoked potentials [39] and a possible VEP-habituation deficit interictally in migraine [40]. However, neither hyperexcitability, nor the preferred concept of "hyperresponsitivity" [41], have been confirmed in later blinded studies of interictal VEP [42, 43].

Cosentino et al. [23] found normal preictal potentiation of motor evoked potential trains ( $5 \mathrm{~Hz}$ MEP) in migraine while flat or inhibitory patterns were observed in interictal, ictal and postictal phases. This MEPpotentiation is thought to be mediated by cortical shortterm synaptic enhancement mainly due to calciumdependent regulation of glutamate release. Their results were interpreted as high (i.e. normal) threshold for inhibitory homeostatic responses in the preictal phase, due to low motor cortex activity. [23]. The same group suggested hyperresponsivity of excitatory intracortical circuits as the pathogenesis of migraine [44]. Compensatory effects such as lower thresholds for inhibitory regulation and increased "top-down" inhibitory control could explain normal interictal cortical activation despite this underlying cortical hyperresponsivity [44]. Destabilization of this excitatory/inhibitory balance could be the precipitating factor triggering the migraine attack. Decrease in cortical activation observed as increased beta activity in this study may cause higher thresholds for inhibitory homeostatic plasticity in the preictal phase as seen in the mentioned study of MEPpotentiation [23, 44]. Consequently, reduced inhibitory control of the suggested primitive hyperresponsivity might be represented as increased preictal beta-ERD as seen in this study.

In our previous article we found increased preictal PMBS in the ipsilateral sensorimotor cortex [14], suggesting increased inhibition 1-3 s after the termination of movement. Interneurons receiving facilitatory stimuli from interhemispheric projections [14, 45-47] may also be involved because PMBS-changes was mostly ipsilateral [13]. Hence, the combined results of increased preictal baseline-beta, beta-ERD and PMBS suggest that the sensorimotor cortex may undergo an increased deactivation-activation-deactivation cycle within the few seconds before, during and after the sensorimotor movement task. This may reflect an instability of thalamocortical, interhemispheric and intracortical regulatory mechanisms in the preictal phase.

Preictal cortical deactivation is in concert with recent findings of reduced baseline pain scores that suggested enhanced preictal endogenous analgesia [1]. Reduced habituation to electrically induced pain [48] and tonic heat pain [1] has been shown in the preictal phase of migraine, suggesting hyperresponsivity to persistent painful stimulation. Also, Marciszewski et al. found decreased pain sensitivity combined with greater activation of spinal trigeminal nucleus during noxious orofacial stimulation in the period immediately before a migraine attack [49].

Thalamus is probably also important in mediation of dysfunctional pain modulation in migraine, at least between attacks [50-52]. Our results show that there also is increased responsivity during non-noxious sensory stimuli at a thalamocortical level in the preictal phase. Meylakh et al. [53] recently reported increased power of infra-slow fMRI-oscillations in thalamus, PAG and hypothalamus immediately prior to the migraine attack. These findings hypothesized to reflect increased amplitude and synchrony of astrocyte calcium waves are difficult to compare with results 
based on electrophysiological recordings. However, recent longitudinal fMRI studies also suggest that a hypothalamic-brainstem network dysfunction may be relevant during the preictal phase of migraine [11].

We did not detect significant differences in baseline beta power or beta-ERD between migraineurs in the interictal phase and control subjects. Reduced interictal cortical pre-activation has been proposed previously, mainly based on a non-significant trend towards lower first-block visual evoked potential (VEP) amplitude in migraineurs $[40,54,55]$. However, first-block VEP amplitude is normal in most studies [42, 43]. Hence, neither previous VEP nor present beta-ERD results support this theory for the interictal phase in migraine. Furthermore, the theory of cortical hyperresponsivity compensated interictally by inhibitory control in migraine could be an explanation of non-significant interictal analyses. However, it is also possible that increased pre-activation variability is present interictally, explaining why results differ between studies.

\section{Strengths and limitations of the study}

It is possible that many of the neurophysiological changes in the migraine brain are subtle, needing higher-powered studies to be consistently observed. Furthermore, a great challenge in evaluating the migraine cycle is to conduct longitudinal studies with enough subjects for paired recordings, and a greater number of subjects with more test repetitions can detect smaller changes. Small groups for paired analyses may have contributed to the possibility of type II errors in this study. However, a blinded, longitudinal study design such as in the present study is important to investigate the very phase specific alterations of migraine pathophysiology [56].

Furthermore, healthy subjects were not screened for relatives with migraine, and a few controls might in theory be susceptible to migraine and to VEP-potentiation [57]. Moreover, in a previous sensitivity analysis the removal of controls with migraine relatives did not affect the VEP habituation results [43]. This potential confounder may possibly contribute to the risk of type II errors, but it should not affect present positive beta-ERD findings.

Scalp electrical recordings is known to be contaminated by contributions of electromyography, possibly from activation of scalp and neck muscles [58]. The present study did not utilise specific methodology for reducing such artefacts. However, EMG contamination is mostly shown for frequencies above $20 \mathrm{~Hz}$ which is higher than the frequency band chosen for our beta power analyses [58].

In studies of event related dynamics, it is important to differentiate between self-paced and triggered movement as neurophysiological changes may be detected before the movement itself when there is a planning phase [29]. All movements in this study was triggered to limit the uncertainty in determining start of relevant cortical activity during a planning phase.

As in our previous study [14], an a priori methodological selection of the interval $12-19 \mathrm{~Hz}$ as beta band $[35,36]$ was conducted to avoid type I errors. However, other studies on beta event related dynamics have yielded results for different cut-offs for beta band frequencies [59-62]. It is possible that identical oscillatory processes are reflected by slightly different beta band frequencies between subjects. Such interpersonal differences may affect results from two-sample statistics comparing groups, however the significant findings in this study was found for longitudinal analyses of the same subjects. Furthermore, more recent findings indicate that movement-related beta oscillations show high intraindividual reliability and are well suited to detect individual differences in a longitudinal study design [63].

The present ERD-calculation method does not differentiate between phase-locked and non-phaselocked activity [27], but this is seemingly not necessary for movement related beta-alterations [64]. For future studies it would be of interest also to try more advanced time-frequency domain methods like event related spectral perturbation (ERSP) also for movement related ERD-paradigms [65].

\section{Conclusion}

We were able to show cyclic fluctuation of cortical sensory processing in migraine patients through alterations in beta-ERD and baseline beta power. In the $36-h$ period before headache, there seems to be a decrease in cortical pre-activation and an increase in the relative stimulusinduced response. Altered pre-activation may lead to changes in thresholds for inhibitory responses and consequently increased beta-ERD response, possibly reflecting a generally increased cortical responsivity in migraine. We did not find this central sensory fluctuation during the ictal phase, but the study was not powered to perform direct comparisons between the preictal and the ictal phase. Cyclic alterations in the activity of second- and third-order afferent somatosensory neurons, and their associated cortical and/or thalamic interneurons, may accordingly be a central part of the migraine pathophysiology. Combined investigations of cyclic fluctuations in event related responses (ERD/ERS) and targeted transcranial magnetic stimulation (TMS) paradigms should be conducted to understand the involvement of different excitatory and inhibitory pathways in migraine pathophysiology. 


\section{Abbreviations}

CNS: Central Nervous System; ECG: Electrocardiography;

EEG: Electroencephalography; EMG: Electromyography; ERD: Event-related desynchronization; ERS: Event-related synchronization; GABA: GammaAminobutyric acid; M: Motor test; MEP: Motor evoked potentials; NMDA: NMethyl-D-aspartate; PAG: Periaqueductal gray; PMBS: Post-movement beta synchronization; R-ANOVA: Repeated measures ANOVA; SM: Sensorimotor test; TMS: Transcranial Magnetic Stimulation; VEP: Visual evoked potentials

\section{Acknowledgements}

The authors are most grateful for the extensive effort and contribution by both migraine patients and controls as well as for the assistance from Grethe Helde, Gøril Bruvik Gravdahl, Knut Hagen and Lars Jacob Stovner.

\section{Authors contributions}

MSM performed the analyses, main interpretations of the results and did most of the manuscript preparations. TS and MHB planned the original study and collected data. TS, MHB, PMO and MU performed major contributions to interpretation of the results and preparations of the article. MS was essential for planning and implementation of the study and collection of data. All authors read and approved the final manuscript.

\section{Funding}

The present study had no external funding.

\section{Availability of data and materials}

The datasets used and analysed during the current study are available from the corresponding author on reasonable request.

\section{Ethics approval and consent to participate}

We obtained written consent from all the subjects. The regional ethical committee approved of the study.

\section{Consent for publication}

Not applicable.

\section{Competing interests}

All authors declare no conflict of interest regarding this study and article.

\section{Author details}

${ }^{1}$ Department of Neuromedicine and Movement Science, Faculty of Medicine and Health Sciences, NTNU - Norwegian University of Science and Technology, Trondheim, Norway. ${ }^{2}$ Department of Clinical Medicine, University of Bergen, Bergen, Norway. ${ }^{3}$ Department of Neurology, Haukeland University Hospital, Bergen, Norway. ${ }^{4}$ Department of Neurology and Clinical Neurophysiology, St. Olavs Hospital, Trondheim, Norway.

\section{Received: 24 January 2019 Accepted: 17 June 2019}

Published online: 09 July 2019

\section{References}

1. Uglem M, Omland PM, Nilsen KB, Tronvik E, Stovner LJ, Hagen K et al (2017) Does pain sensitivity change by migraine phase? A blinded longitudinal study. Cephalalgia. 37(14):1337-1349

2. Sand T, Zhitniy N, Nilsen KB, Helde G, Hagen K, Stovner LJ (2008) Thermal pain thresholds are decreased in the migraine preattack phase. Eur J Neurol 15(11):1199-1205

3. Laurell K, Artto V, Bendtsen L, Hagen K, Haggstrom J, Linde M et al (2016) Premonitory symptoms in migraine: a cross-sectional study in 2714 persons. Cephalalgia. 36(10):951-959

4. Pascual J, Quintela E, Cuvellier JC, Mars A, Vallee L (2010) Premonitory symptoms in migraine patients. Cephalalgia. 30(5):639 author reply -40

5. Quintela E, Castillo J, Munoz P, Pascual J (2006) Premonitory and resolution symptoms in migraine: a prospective study in 100 unselected patients. Cephalalgia. 26(9):1051-1060

6. Maniyar FH, Sprenger T, Monteith T, Schankin CJ, Goadsby PJ (2015) The premonitory phase of migraine--what can we learn from it? Headache. 55(5):609-620

7. Giffin NJ, Ruggiero L, Lipton RB, Silberstein SD, Tvedskov JF, Olesen J et al (2003) Premonitory symptoms in migraine: an electronic diary study. Neurology. 60(6):935-940
8. Goadsby PJ, Holland PR, Martins-Oliveira M, Hoffmann J, Schankin C, Akerman S (2017) Pathophysiology of migraine: a disorder of sensory processing. Physiol Rev 97(2):553-622

9. Karsan N, Goadsby PJ (2018) Biological insights from the premonitory symptoms of migraine. Nat Rev Neurol

10. Maniyar FH, Sprenger T, Monteith T, Schankin C, Goadsby PJ (2014) Brain activations in the premonitory phase of nitroglycerin-triggered migraine attacks. Brain. 137(Pt 1:232-241

11. Schulte LH, May A (2016) The migraine generator revisited: continuous scanning of the migraine cycle over 30 days and three spontaneous attacks. Brain. 139(Pt 7:1987-1993

12. Siniatchkin M, Averkina N, Andrasik F, Stephani U, Gerber WD (2006) Neurophysiological reactivity before a migraine attack. Neurosci Lett 400(1-2):121-124

13. Fritzer G, Strenge H, Goder R, Gerber WD, Aldenhoff J (2004) Changes in cortical dynamics in the preictal stage of a migraine attack. J Clin Neurophysiol 21(2):99-104

14. Mykland MS, Bjork MH, Stjern M, Sand T (2018) Alterations in postmovement beta event related synchronization throughout the migraine cycle: a controlled, longitudinal study. Cephalalgia. 38(4):718-729

15. Goder R, Fritzer G, Kapsokalyvas A, Kropp P, Niederberger U, Strenge H et al (2001) Polysomnographic findings in nights preceding a migraine attack. Cephalalgia. 21(1):31-37

16. Judit A, Sandor PS, Schoenen J (2000) Habituation of visual and intensity dependence of auditory evoked cortical potentials tends to normalize just before and during the migraine attack. Cephalalgia. 20(8):714-719

17. Sand T, Zhitniy N, White LR, Stovner LJ (2008) Visual evoked potential latency, amplitude and habituation in migraine: a longitudinal study. Clin Neurophysiol 119(5):1020-1027

18. Bjork M, Hagen K, Stovner L, Sand T (2011) Photic EEG-driving responses related to ictal phases and trigger sensitivity in migraine: a longitudinal, controlled study. Cephalalgia. 31(4):444-455

19. Bjork M, Stovner LJ, Hagen K, Sand T What initiates a migraine attack? Conclusions from four longitudinal studies of quantitative EEG and steadystate visual-evoked potentials in migraineurs. Acta Neurol Scand Suppl 2011(191):56-63

20. Bjørk MH, Sand T (2008) Quantitative EEG power and asymmetry increase $36 \mathrm{~h}$ before a migraine attack. Cephalalgia.

21. Burstein R, Jakubowski M, Garcia-Nicas E, Kainz V, Bajwa Z, Hargreaves R et al (2010) Thalamic sensitization transforms localized pain into widespread allodynia. Ann Neurol 68(1):81-91

22. Amin FM, Hougaard A, Magon S, Sprenger T, Wolfram F, Rostrup E et al (2017) Altered thalamic connectivity during spontaneous attacks of migraine without aura: a resting-state fMRI study. Cephalalgia.: 333102417729113

23. Cosentino G, Fierro B, Vigneri S, Talamanca S, Paladino P, Baschi R et al (2014) Cyclical changes of cortical excitability and metaplasticity in migraine: evidence from a repetitive transcranial magnetic stimulation study. Pain. 155(6):1070-1078

24. Siniatchkin M, Gerber WD, Kropp P, Vein A (1999) How the brain anticipates an attack: a study of neurophysiological periodicity in migraine. Funct Neurol 14(2):69-77

25. Stankewitz A, May A (2007) Cortical excitability and migraine. Cephalalgia. 27(12):1454-1456

26. Neuper C, Wortz M, Pfurtscheller G (2006) ERD/ERS patterns reflecting sensorimotor activation and deactivation. Prog Brain Res 159:211-222

27. Pfurtscheller $G$ (2001) Functional brain imaging based on ERD/ERS. Vis Res 41(10-11):1257-1260

28. Takemi M, Masakado Y, Liu M, Ushiba J (2015) Sensorimotor event-related desynchronization represents the excitability of human spinal motoneurons. Neuroscience. 297:58-67

29. Pfurtscheller G, Lopes da Silva FH (1999) Event-related EEG/MEG synchronization and desynchronization: basic principles. Clin Neurophysiol 110(11):1842-1857

30. Nakayashiki K, Saeki M, Takata Y, Hayashi Y, Kondo T (2014) Modulation of event-related desynchronization during kinematic and kinetic hand movements. J Neuroeng Rehabil 11:90

31. Fallon N, Chiu YH, Li X, Nurmikko TJ, Stancak A (2013) Ipsilateral cortical activation in fibromyalgia patients during brushing correlates with symptom severity. Clin Neurophysiol 124(1):154-163 
32. Bjork MH, Stovner LJ, Nilsen BM, Stjern M, Hagen K, Sand T (2009) The occipital alpha rhythm related to the "migraine cycle" and headache burden: a blinded, controlled longitudinal study. Clin Neurophysiol 120(3):464-471

33. Bjork MH, Stovner LJ, Engstrom M, Stjern M, Hagen K, Sand T (2009) Interictal quantitative EEG in migraine: a blinded controlled study. J Headache Pain 10(5):331-339

34. Klem GH, Luders HO, Jasper HH, Elger C (1999) The ten-twenty electrode system of the international federation. The International Federation of Clinical Neurophysiology. Electroencephalogr Clin Neurophysiol Suppl 52:3-6

35. Pfurtscheller G, Neuper C, Pichler-Zalaudek K, Edlinger G, Lopes da Silva FH (2000) Do brain oscillations of different frequencies indicate interaction between cortical areas in humans? Neurosci Lett 286(1):66-68

36. Neuper C, Pfurtscheller G (2001) Evidence for distinct beta resonance frequencies in human EEG related to specific sensorimotor cortical areas. Clin Neurophysiol 112(11):2084-2097

37. Leocani L, Toro C, Manganotti P, Zhuang P, Hallett M (1997) Event-related coherence and event-related desynchronization/synchronization in the 10 $\mathrm{Hz}$ and $20 \mathrm{~Hz}$ EEG during self-paced movements. Electroencephalogr Clin Neurophysiol 104(3):199-206

38. Stancak A Jr, Pfurtscheller G (1996) Event-related desynchronisation of central beta-rhythms during brisk and slow self-paced finger movements of dominant and nondominant hand. Brain Res Cogn Brain Res 4(3):171-183

39. Hegerl U, Juckel G (1993) Intensity dependence of auditory evoked potentials as an indicator of central serotonergic neurotransmission: a new hypothesis. Biol Psychiatry 33(3):173-187

40. Schoenen J (1996) Deficient habituation of evoked cortical potentials in migraine: a link between brain biology, behavior and trigeminovascular activation? Biomed Pharmacother 50(2):71-78

41. Coppola G, Pierelli F, Schoenen J (2007) Is the cerebral cortex hyperexcitable or hyperresponsive in migraine? Cephalalgia. 27(12):1427-1439

42. Omland PM, Nilsen KB, Uglem M, Gravdahl G, Linde M, Hagen K et al (2013) Visual evoked potentials in interictal migraine: no confirmation of abnormal habituation. Headache. 53(7):1071-1086

43. Omland PM, Uglem M, Hagen K, Linde M, Tronvik E, Sand T (2016) Visua evoked potentials in migraine: is the "neurophysiological hallmark" concept still valid? Clin Neurophysiol 127(1):810-816

44. Cosentino G, Fierro B, Brighina F (2014) From different neurophysiological methods to conflicting pathophysiological views in migraine: a critical review of literature. Clin Neurophysiol 125(9):1721-1730

45. Schnitzler A, Kessler KR, Benecke R (1996) Transcallosally mediated inhibition of interneurons within human primary motor cortex. Exp Brain Res 112(3):381-391

46. van Wijk BC, Beek PJ, Daffertshofer A (2012) Differential modulations of ipsilateral and contralateral beta (de) synchronization during unimanual force production. Eur J Neurosci 36(1):2088-2097

47. Daffertshofer A, Peper CL, Beek PJ (2005) Stabilization of bimanual coordination due to active interhemispheric inhibition: a dynamical account. Biol Cybern 92(2):101-109

48. Strupf M, Fraunberger B, Messlinger K, Namer B (2018) Cyclic changes in sensations to painful stimuli in migraine patients. Cephalalgia: 333102418793641

49. Marciszewski KK, Meylakh N, Di Pietro F, Mills EP, Macefield VG, Macey PM et al (2018) Changes in brainstem pain modulation circuitry function over the migraine cycle. J Neurosci

50. Younis S, Hougaard A, Noseda R, Ashina M Current understanding of thalamic structure and function in migraine. Cephalalgia. $0(0)$ : 0333102418791595

51. Coppola G, Bracaglia M, Di Lenola D, lacovelli E, Di Lorenzo C, Serrao M et al (2016) Lateral inhibition in the somatosensory cortex during and between migraine without aura attacks: correlations with thalamocortical activity and clinical features. Cephalalgia. 36(6):568-578

52. Porcaro C, Di Lorenzo G, Seri S, Pierelli F, Tecchio F, Coppola G (2017) Impaired brainstem and thalamic high-frequency oscillatory EEG activity in migraine between attacks. Cephalalgia. 37(10):915-926

53. Meylakh N, Marciszewski KK, Di Pietro F, Macefield VG, Macey PM, Henderson LA (2018) Deep in the brain: changes in subcortical function immediately preceding a migraine attack. Hum Brain Mapp 39(6):2651-2663
54. de Tommaso M, Ambrosini A, Brighina F, Coppola G, Perrotta A, Pierelli F et al (2014) Altered processing of sensory stimuli in patients with migraine. Nat Rev Neurol 10(3):144-155

55. Schoenen J, Wang W, Albert A, Delwaide PJ (1995) Potentiation instead of habituation characterizes visual evoked potentials in migraine patients between attacks. Eur J Neurol 2(2):115-122

56. Peng KP, May A (2018) Quantitative sensory testing in migraine patients must be phase-specific. Pain. 159(11):2414-2416

57. Lisicki M, Ruiz-Romagnoli E, D'Ostilio K, Piedrabuena R, Giobellina R, Schoenen J et al (2017) Familial history of migraine influences habituation of visual evoked potentials. Cephalalgia. 37(11):1082-1087

58. Whitham EM, Pope KJ, Fitzgibbon SP, Lewis T, Clark CR, Loveless S et al (2007) Scalp electrical recording during paralysis: quantitative evidence that EEG frequencies above $20 \mathrm{~Hz}$ are contaminated by EMG. Clin Neurophysiol 118(8):1877-1888

59. Houdayer E, Labyt E, Cassim F, Bourriez JL, Derambure P (2006) Relationship between event-related beta synchronization and afferent inputs: analysis of finger movement and peripheral nerve stimulations. Clin Neurophysiol 117(3):628-636

60. Kuhn AA, Doyle L, Pogosyan A, Yarrow K, Kupsch A, Schneider GH et al (2006) Modulation of beta oscillations in the subthalamic area during motor imagery in Parkinson's disease. Brain. 129(Pt 3:695-706

61. Bizovicar N, Dreo J, Koritnik B, Zidar J (2014) Decreased movement-related beta desynchronization and impaired post-movement beta rebound in amyotrophic lateral sclerosis. Clin Neurophysiol 125(8):1689-1699

62. Meirovitch Y, Harris H, Dayan E, Arieli A, Flash T (2015) Alpha and beta band event-related desynchronization reflects kinematic regularities. J Neurosci 35(4):1627-1637

63. Espenhahn S, de Berker AO, van Wijk BCM, Rossiter HE, Ward NS (2017) Movement-related beta oscillations show high intra-individual reliability. Neuroimage. 147:175-185

64. Kalcher J, Pfurtscheller G (1995) Discrimination between phase-locked and non-phase-locked event-related EEG activity. Electroencephalogr Clin Neurophysiol 94(5):381-384

65. Wang BA, Viswanathan S, Abdollahi RO, Rosjat N, Popovych S, Daun S et al (2017) Frequency-specific modulation of connectivity in the ipsilateral sensorimotor cortex by different forms of movement initiation. Neuroimage. 159:248-260

\section{Publisher's Note}

Springer Nature remains neutral with regard to jurisdictional claims in published maps and institutional affiliations.

Ready to submit your research? Choose BMC and benefit from:

- fast, convenient online submission

- thorough peer review by experienced researchers in your field

- rapid publication on acceptance

- support for research data, including large and complex data types

- gold Open Access which fosters wider collaboration and increased citations

- maximum visibility for your research: over $100 \mathrm{M}$ website views per year

At BMC, research is always in progress.

Learn more biomedcentral.com/submissions 